\title{
A margin adaptive scheduling algorithm for FBMC/OQAM systems
}

\author{
Màrius Caus*, Ana I. Perez-Neira ${ }^{\dagger *}$, Marco Moretti ${ }^{\ddagger}$ and Adrian Kliks ${ }^{\S}$ \\ *Centre Tecnològic de Telecomunicacions de Catalunya (CTTC), Castelldefels, Barcelona, Spain \\ ${ }^{\dagger}$ Dept. of Signal Theory and Communications Universitat Politècnica de Catalunya (UPC), Barcelona, Spain \\ ${ }_{\ddagger}^{\ddagger}$ Dept. of Information Engineering University of Pisa (UP), Pisa, Italy \\ ${ }^{\S}$ Chair of Wireless Communications, Poznan University of Technology (PUT), Poznan, Poland
}

\begin{abstract}
This paper addresses the margin adaptive problem for the filter bank multicarrier (FBMC) modulation based on the offset QAM (OQAM), referred to as FBMC/OQAM. The analysis conducted in this paper reveals that due to the specific FBMC/OQAM transmission format the system has to deal with inter-user, inter-symbol and inter-carrier interference to solve the resource allocation problem. To manage the interference, the successive channel allocation method, originally proposed for the orthogonal frequency division multiplexing (OFDM), has been modified by organizing subcarriers in groups and assigning subcarriers to users in a block-wise fashion. Following this strategy and making some approximations we demonstrate that it is possible to pose a single problem that is valid for OFDM and FBMC/OQAM. In this case, numerical results show that FBMC/OQAM is more energy-efficient than OFDM because it requires less power to transmit the same information rate.
\end{abstract}

\section{INTRODUCTION}

The orthogonal frequency division multiplexing (OFDM) has been the modulation of choice in various communication systems. Its value stems from the fact that frequency selective channels can be modeled as a set of parallel flat fading channels. However, this merit comes with the cost of transmitting redundancy in the form of a cyclic prefix (CP) and shaping the subcarrier signals with the rectangular window. As a consequence, OFDM exhibits poor stopband attenuation. A viable alternative to OFDM is the filter bank multicarrier (FBMC) modulation based on the offset QAM (OQAM), referred to as FBMC/OQAM or OFDM/OQAM [1], which does not transmit the $\mathrm{CP}$ and shapes subcarriers with well-frequency-localized waveforms. The price that is paid to not transmit redundancy is a weak orthogonality that it is only satisfied in the real domain. This is the main obstacle to extend FBMC/OQAM to multiple-input-multiple-output (MIMO) architectures [2], which is crucial to enhance the performance. Knowing that the solutions devised for OFDM cannot be applied to FBMC/OQAM in general, several techniques have been proposed to combine MIMO and FBMC/OQAM [3].

One of the few works that addresses the resource allocation problem in the FBMC/OQAM context is presented in [4], where the rate in the multiple access channel is maximized given power and users' rate constraints. In [5] the authors seek for maximizing the downlink capacity of cognitive radio systems. By contrast, the work presented in this paper aims at minimizing the transmit power in the downlink subject to users' rate constraints. The propagation conditions considered

978-1-4799-5863-4/14/\$31.00 (c) 2014 IEEE in previous works are such that the interference can be neglected [5], or cancelled out by applying the same signal processing techniques as in OFDM [4]. This is not the case in this paper, where the channel is more frequency selective revealing that the signal received by each user is affected by inter-user interference if the user allocation is different in adjacent subcarriers. To overcome this issue we propose to assign subcarriers to users in a block-wise fashion. Then, we demonstrate that FBMC/OQAM systems can benefit from the algorithms proposed in [6] to solve the margin adaptive problem. It is important to remark that this paper focuses on multiuser multiple-input-single-output (MU-MISO) communication systems, because in this configuration FBMC/OQAM exhibits robustness against the frequency selectivity, while it achieves the same spatial channels gains as OFDM [7].

The main contribution of this paper consists in modifying the scheduling algorithm presented in [6] according to the FBMC/OQAM transmission scheme, by proposing a specific subcarrier grouping and taking into account the existence of inter-user, inter-symbol and inter-carrier interference.

Next section presents the MU-MISO system model. Section III discusses the concept of block diagonalization. Section IV presents the margin adaptive problem and Section $\mathrm{V}$ describes the successive channel allocation. Simulation results and conclusions are included in sections VI and VII, respectively.

\section{SYSTEM MODEL}

Consider the downlink of a MU-MISO communication system where the terminals and the base station (BS) are equipped with a single and $N_{T}$ antennas, respectively. The BS uses the spatial dimension to simultaneously serve up to $N_{U}$ users in the same frequency resources. When the FBMC/OQAM transceiver is considered, the signal received by the $l$ th user after demodulating the $q$ th subcarrier is [7]:

$$
\begin{gathered}
y_{l q}[k]=\sum_{\substack{u=1 \\
N_{U}}}^{q+1} \sum_{m=q-1}^{3} \alpha_{\tau=-3}[\tau] \mathbf{H}_{l m} \mathbf{B}_{u m} \\
\boldsymbol{H}_{m}[k-\tau] d_{u m}[k-\tau]+w_{l q}[k] \\
\theta_{m}[k]=\left[\begin{array}{lll}
H_{l 1}(m) & \ldots & \left.H_{l N_{T}}(m)\right] \\
j & k+m & \text { even }
\end{array}\right.
\end{gathered}
$$

for $0 \leq q \leq M-1$ and $1 \leq l \leq N_{U}$. Let $w_{l q}[k]$ be the noise that contaminates the reception of the $l$ th user on the $q$ th subcarrier, which follows this distribution $w_{l q}[k] \sim \mathcal{C N}\left(0, N_{0}\right)$. 
Table I. INTRINSIC INTERFERENCES UNDER IDEAL PROPAGATION CONDITIONS

\begin{tabular}{|c|c|c|c|c|c|c|c|}
\hline & $\mathrm{k}=-3$ & $\mathrm{k}=-2$ & $\mathrm{k}=-1$ & $\mathrm{k}=0$ & $\mathrm{k}=1$ & $\mathrm{k}=2$ & $\mathrm{k}=3$ \\
\hline$\alpha_{q q-1}[k]$ & $-\mathrm{j} 0.0429$ & -0.1250 & $\mathrm{j} 0.2058$ & 0.2393 & $-\mathrm{j} 0.2058$ & -0.1250 & $\mathrm{j} 0.0429$ \\
\hline$\alpha_{q q}[k]$ & -0.0668 & 0 & 0.5644 & 1 & 0.5644 & 0 & -0.0668 \\
\hline$\alpha_{q q+1}[k]$ & $\mathrm{j} 0.0429$ & -0.1250 & $-\mathrm{j} 0.2058$ & 0.2393 & $\mathrm{j} 0.2058$ & -0.1250 & $-\mathrm{j} 0.0429$ \\
\hline
\end{tabular}

The channel frequency response is assumed flat at the subcarrier level. In this sense, the term $\mathbf{H}_{l m} \in \mathbb{C}^{1 \times N_{T}}$ denotes the MISO channel frequency response seen by the $l$ th user on the $m$ th subcarrier. The sequence $d_{u m}[k]$ is frequency multiplexed on the $m$ th subcarrier and it contains real-valued PAM symbols that are intended for the $u$ th user. Note that $d_{u m}[k]$ is linearly precoded with $\mathbf{B}_{u m} \in \mathbb{C}^{N_{T} \times 1}$. The coefficients $\left\{\alpha_{q m}[k]\right\}$ denote the intrinsic interference and they are defined as

$$
\begin{aligned}
\alpha_{q m}[k] & =\left(f_{m}[n] * f_{q}^{*}[-n]\right)_{\downarrow \frac{M}{2}} \\
f_{m}[n] & =p[n] e^{j \frac{2 \pi}{M} m\left(n-\frac{L-1}{2}\right)},
\end{aligned}
$$

where $*$ denotes convolution. Note that $f_{m}[n]$ is the subband pulse that is obtained by frequency shifting the low-pass prototype pulse $p[n]$ the length of which is $L$. In this work we opt for the design described in [8] with an overlapping factor equal to four. The operation $(.)_{\downarrow x}$ performs a decimation by a factor of $x$. In the $q$ even case $\left\{\alpha_{q m}[k]\right\}$ takes the values gathered in Table I. The same magnitudes hold when $q$ is odd but the sign may vary.

To get rid of the interferences $y_{l q}[k]$ is post-processed as follows:

$$
\begin{aligned}
& \check{d}_{l q}[k]=\Re\left(\theta_{q}^{*}[k] a_{l q} y_{l q}[k]\right)=a_{l q} \Re\left(\mathbf{H}_{l q} \mathbf{B}_{l q}\right) d_{l q}[k]+ \\
& \sum_{\substack{(m, \tau, u) \neq \\
(q, 0, l) \\
+a_{l q} \Re\left(\theta_{q}^{*}[k] w_{l q}[k]\right) .}} a_{l q} \Re\left(\theta_{q}^{*}[k] \theta_{m}[k-\tau] \alpha_{q m}[\tau] \mathbf{H}_{l m} \mathbf{B}_{u m}\right) d_{u m}[k-\tau]
\end{aligned}
$$

Note that the equalizer $a_{l q}$ is constrained to be real-valued [7].

\section{SDMA WITH BLOCK DIAGONALIZATION}

One solution to achieve spatial division multiple access (SDMA) consists in following the block diagonalization (BD) approach [9]. Although the BD technique succeeds in achieving interference-free data multiplexing, it imposes $N_{T} \geq N_{U}$ in MU-MISO communication systems. It can be checked that the subband processing proposed in [7] guarantees that (6) is free of inter-symbol interference (ISI), inter-carrier interference (ICI) and inter-user interference (IUI), as long as the users served on a given subcarrier are the same on adjacent subcarriers. Then, the maximum achievable rate becomes

$$
\begin{gathered}
r_{l q}=\frac{1}{2} \log _{2}\left(1+S I N R_{l q}\right) \\
S I N R_{l q}=p_{l q}\left\|\mathbf{H}_{l q} \check{\mathbf{V}}_{l q}^{0}\right\|_{2}^{2} / 0.5 N_{0}=p_{l q} \lambda_{l q},
\end{gathered}
$$

where $\check{\mathbf{V}}_{l q}^{0} \in \mathbb{C}^{N_{T} \times N_{T}-N_{U}+1}$ spans the null space of

$$
\check{\mathbf{H}}_{l q}=\left[\begin{array}{llll}
\mathbf{H}_{1 q}^{T} & \cdots \mathbf{H}_{l-1 q}^{T} & \mathbf{H}_{l+1 q}^{T} \cdots \mathbf{H}_{N_{U} q}^{T}
\end{array}\right]^{T} .
$$

Note that the power allocated to $d_{l q}[k]$ is given by $p_{l q}$. The factor $\frac{1}{2}$ in (7) has to do with the fact that the variables in (6) are real-valued. To get (7) we assume a continuous transmission, so that the tails of the pulse have no impact on the rate. This does not hold true in burst-like transmission, but we leave this case for future work. In the OFDM counterpart the rate is given by (7) but the factor $\frac{1}{2}$ is dropped because the information is conveyed in both the in-phase and quadrature components, i.e. the rate is formulated as

$$
r_{l q}=\log _{2}\left(1+2 p_{l q}\left\|\mathbf{H}_{l q} \check{\mathbf{V}}_{l q}^{0}\right\|_{2}^{2} / N_{0}\right) .
$$

The SINR is not modified but there are two aspects that have to be taken into account. The first one is that the power of the desired signal is multiplied by two since the symbols transmitted in OFDM are drawn from the QAM scheme and the real-valued symbols $\left\{d_{l q}[k]\right\}$ are obtained from either the real or the imaginary parts of the QAM constellation points. The second relevant aspect has to do with the fact that the power of the noise is not halved because detection is performed in the complex domain. Let us stress that when the power coefficients, the bandwidth and the sampling frequency are kept unchanged in both modulations, then the rate expressed in bps is the same in FBMC/OQAM and OFDM without CP.

To ease the comparison between FBMC/OQAM and OFDM we consider the rate that corresponds to one OFDM symbol period. Thus, the rate that will be used from this point on unless otherwise stated is expressed in this form

$$
r_{l q}=\log _{2}\left(1+S I N R_{l q}\right),
$$

which is valid for both modulations. Bearing in mind that (11) is achieved in FBMC/OQAM systems by transmitting two multicarrier symbols, it follows that the total transmitted power in one OFDM symbol period is $\sum_{l=1}^{N_{U}} \sum_{q=0}^{M-1} 2 p_{l q}$. By contrast, taking into account that the symbols in OFDM are obtained from a complex-valued constellation diagram, the transmitted power in OFDM is equal to $\sum_{l=1}^{N_{U}} \sum_{q=0}^{M-1}\left(1+\frac{L_{C P}}{M}\right) 2 p_{l q}$, where $L_{C P}$ denotes the CP length. For the sake of clarity the $\mathrm{CP}$ will be only considered when the transmitted power is computed and will be neglected when the rate is evaluated.

\section{MARGIN ADAPTIVE SCHEDULING ALGORITHM}

It is worth emphasizing that when $N_{T}<N_{U}$ the BS selects $N_{T}$ out of $N_{U}$ users over each subcarrier. However, the user assignment has to remain constant over all subbands so that the BD approach is able to remove the ICI in FBMC/OQAM. This can be easily proved as follows. Consider a toy example where 2 users are allocated in each subcarrier. Imagine that subcarriers $\{q-1, q+1\}$ are assigned to the users $u_{1}$ and $u_{2}$, while the users $l_{1}$ and $l_{2}$ are allocated in the $q$ th subcarrier. If $\left\{a_{l q}\right\}$ and $\left\{\mathbf{B}_{l q}\right\}$ are designed according to [7], we get

$$
\begin{aligned}
& \check{d}_{l_{i} q}[k]=a_{l_{i} q} \Re\left(\mathbf{H}_{l_{i} q} \mathbf{B}_{l_{i} q}\right) d_{l_{i} q}[k]+ \\
& \sum_{\tau=-3}^{3} \sum_{m=q-1}^{q+1} \sum_{j=1}^{2} a_{l_{i}} \Re\left(\theta_{q}^{*}[k] \theta_{m}[k-\tau] \alpha_{q m}[\tau] \mathbf{H}_{l_{i} m} \mathbf{B}_{u_{j} m}\right) \\
& \times d_{u_{j} m}[k-\tau]+a_{l_{i} q} \Re\left(\theta_{q}^{*}[k] w_{l_{i} q}[k]\right),
\end{aligned}
$$


for $i=1,2$. Note that in (12) there is no contribution from user $l_{j}$ for $j \neq i$. On the negative side, we cannot neglect the ICI because the precoding vectors do not satisfy $\mathbf{H}_{l_{i} m} \mathbf{B}_{u_{j} m}=\mathbf{0}$. Hence, (12) shows the situations that should be avoided to get rid of the interference. To guarantee that all users achieve a certain rate in the absence of interference, we propose to assign subcarriers to users in a block-wise fashion. In other words, the band is partitioned into $X$ subsets, where the subset $S_{i}$ encompasses these subcarrier indexes $\left[(i-1) \frac{M}{X}, \ldots, i \frac{M}{X}-1\right]$ assuming that $\frac{M}{X}$ is an integer number. Taking into account that the roll-off factor of the prototype pulse is close to one, the first carrier of each set is left empty to isolate the blocks. Based on that we define $\bar{S}_{i}=S_{i}-\left\{(i-1) \frac{M}{X}\right\}$ for all $i$.

In the light of the above discussion, the optimization problem is posed as follows:

$$
\begin{aligned}
\underset{\left\{p_{l q}\right\},\left\{\rho_{l i}\right\}}{\operatorname{argmin}} & \sum_{l=1}^{N_{U}} \sum_{i=1}^{X} \sum_{q \in \bar{S}_{i}} p_{l q} \\
\text { s.t. } & \sum_{i=1}^{X} \rho_{l i} \sum_{q \in \bar{S}_{i}} \log _{2}\left(1+p_{l q} \lambda_{l q}\right) \geq R_{l}, \quad 1 \leq l \leq N_{U} \\
& \sum_{l=1}^{N_{U}} \rho_{l i} \leq N_{T}, \quad \rho_{l i} \in\{0,1\}, \quad 1 \leq i \leq X .
\end{aligned}
$$

By solving (13) we find the optimal user selection and power allocation, so that the users' rate constraints are guaranteed with the minimum transmit power. Note that the channel gains $\left\{\lambda_{l q}\right\}$ defined in (8) depend on the channel interference matrix, which in turns depend on the subcarrier assignment.

With the aim of substantially reducing the complexity, we assume that constant power is used on each block. Then the number of variables to optimize is significantly reduced and the problem can be simplified as

$$
\begin{array}{ll}
\underset{\left\{p_{l i}\right\},\left\{\rho_{l i}\right\}}{\operatorname{argmin}} & \sum_{l=1}^{N_{U}} \sum_{i=1}^{X}\left|\bar{S}_{i}\right| p_{l i} \\
\text { s.t. } & \sum_{i=1}^{X} \rho_{l i} \sum_{q \in \bar{S}_{i}} \log _{2}\left(1+p_{l i} \lambda_{l q}\right) \geq R_{l}, \quad 1 \leq l \leq N_{U} \\
& \quad \sum_{l=1}^{N_{U}} \rho_{l i} \leq N_{T}, \quad \rho_{l i} \in\{0,1\}, \quad 1 \leq i \leq X,
\end{array}
$$

where $\left|\bar{S}_{i}\right|=\frac{M}{X}-1$ and $p_{l i}$ denotes the power that user $l$ assigns to all subcarriers that belong to $\bar{S}_{i}$. By mapping the sum of rates into a single metric we get more tractable expressions. In this regard, we consider this inequality

$$
\sum_{q \in \bar{S}_{i}} \log _{2}\left(1+p_{l i} \lambda_{l q}\right) \geq\left|\bar{S}_{i}\right| \log _{2}\left(1+p_{l i} \hat{\lambda}_{l i}\right)
$$

where

$$
\hat{\lambda}_{l i}=\left(\prod_{m \in \bar{S}_{i}} \lambda_{l m}\right)^{1 /\left|\bar{S}_{i}\right|}
$$

is the geometric mean [10]. By substituting the sum of rates by the right hand side of (15), we can reformulate (14) as

$$
\begin{array}{ll}
\underset{\left\{p_{l i}\right\},\left\{\rho_{l i}\right\}}{\operatorname{argmin}} \sum_{l=1}^{N_{U}} \sum_{i=1}^{X}\left|\bar{S}_{i}\right| p_{l i} \\
\text { s.t. } \quad \sum_{i=1}^{X} \rho_{l i}\left|\bar{S}_{i}\right| \log _{2}\left(1+p_{l i} \hat{\lambda}_{l i}\right) \geq R_{l}, \quad 1 \leq l \leq N_{U} \\
& \sum_{l=1}^{N_{U}} \rho_{l i} \leq N_{T}, \quad \rho_{l i} \in\{0,1\}, \quad 1 \leq i \leq X .
\end{array}
$$

Now the blocks play the same role as subcarriers and, thus, we can take advantage of existing low-complexity algorithms, see e.g. [6]. The solution of (17) guarantees that the original rate constraints in (13) are satisfied because of the inequality established in (15).

\section{SUCCESSIVE CHANNEL ALLOCATION}

This section focuses on solving (17) by using the linear programming based successive channel allocation (LPSCA) algorithm described in [6]. Among the possible choices we have favored the LPSCA because it exhibits an excellent tradeoff between complexity and performance. The LPSCA was initially though for OFDM systems, which highlights that it may not be easily tailored to the FBMC/OQAM scheme. To cast some light into the applicability of the LPSCA to FBMC/OQAM systems this section briefly describes the algorithm to be employed and the necessary modifications due to the characteristics of the transmitted signal.

The main asset of the LPSCA stems from the reduction of the complexity that is achieved by grouping users into $K_{N_{T}}$ disjoint sets, so that $\left\{1, \ldots, N_{U}\right\}=K_{1} \cup \ldots \cup K_{N_{T}}$. The partitioning is made according to the average channel quality assessment [6]. Then, (17) is also partitioned into $K_{N_{T}}$ independent problems that are sequentially optimized. First we allocate the most distant users to the BS, which means that we start with subset $K_{1}$ and we terminate with the subset $K_{N_{T}}$. When subset $K_{r}$ is addressed, the users that belong to $K_{j}$, for $j<r$ have already been allocated. To guarantee that the rate constraints are not violated, the subband processing is designed to prevent signals that are intended to users in $K_{r}$ from leaking through users that belong to $K_{j}$, for $j<r$. In the rest of the section we focus on the subset $K_{r}$ without loss of generality. In this sense, the signal received by the user $l \in K_{r}$ is

$\check{d}_{l q}[k]=a_{l q} \Re\left(\mathbf{H}_{l q} \mathbf{B}_{l q}\right) d_{l q}[k]+a_{l q}\left(i_{l q}[k]+\Re\left(\theta_{q}^{*}[k] w_{l q}[k]\right)\right)$,

where

$$
\begin{aligned}
i_{l q}[k]= & \sum_{\substack{m=q-1\\
}} \sum_{u \in \mathcal{A}_{m}^{r}} \sum_{\tau=-3}^{3+1} \Re\left(\theta_{q}^{*}[k] \theta_{m}[k-\tau]\right. \\
& \sum_{\substack{q m \\
(m, \tau) \neq(\tau, 0)}} \Re\left(\theta_{q}^{*}[k] \theta_{m}[k-\tau]\right. \\
& \left.\times \alpha_{q m}[\tau] \mathbf{H}_{l m} \mathbf{B}_{l m}\right) d_{l m}[k-\tau] .
\end{aligned}
$$

The already allocated users in the $m$ th subcarrier when the subset $K_{r}$ is addressed, are included in this set $\mathcal{A}_{m}^{r}$ and, thus, $\mathcal{A}_{m}^{r} \subseteq \bigcup_{j=1}^{r-1} K_{j}$. Now the transmit processing is designed to 
project the channel onto the null space of

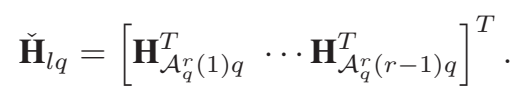

Let $\mathcal{A}_{q}^{r}(j)$ denote the $j$ th element of subset $\mathcal{A}_{q}^{r}$. Bearing (20) in mind, $\mathbf{B}_{l q}$ and $a_{l q}$ are designed as [7] proposes in order to remove the interference, yielding

$$
\begin{gathered}
\mathbf{B}_{l q}=\sqrt{p_{l q}} \frac{\check{\mathbf{U}}_{l q}^{0}\left(\mathbf{H}_{l q} \check{\mathbf{U}}_{l q}^{0}\right)^{H}}{\left\|\check{\mathbf{U}}_{l q}^{0}\left(\mathbf{H}_{l q} \check{\mathbf{U}}_{l q}^{0}\right)^{H}\right\|_{2}} \\
a_{l q}=\frac{\Re\left(\mathbf{H}_{l q} \mathbf{B}_{l q}\right)}{\left|\Re\left(\mathbf{H}_{l q} \mathbf{B}_{l q}\right)\right|^{2}+\sigma_{l q}^{2}},
\end{gathered}
$$

where $\check{\mathbf{U}}_{l q}^{0} \in \mathbb{C}^{N_{T} \times N_{T}-r+1}$ spans the null space of (20). Then, $\Re\left(\theta_{q}^{*}[k] \theta_{m}[k-\tau] \alpha_{q m}[\tau] \mathbf{H}_{l m} \mathbf{B}_{l m}\right)=0$, for $(m, \tau) \neq(q, 0)$. Since the users in $K_{r}$ cannot claim protection against the users in $K_{j}$, for $j<r$, the first term of (19) is not removed and the variance of the noise plus the interference becomes

$$
\begin{gathered}
\sigma_{l q}^{2}=\sum_{\substack{m=q-1\\
}}^{q+1} \sum_{u \in \mathcal{A}_{m}^{r}} \sum_{\tau=-3}^{3} \mid \Re\left(\theta_{q}^{*}[k] \theta_{m}[k-\tau]\right. \\
\left.\times \alpha_{q m}[\tau] \mathbf{H}_{l m} \mathbf{B}_{u m}\right)\left.\right|^{2}+0.5 N_{0} .
\end{gathered}
$$

Considering the values of Table I along with the fact that the same users are allocated in adjacent subcarriers, i.e. $\mathcal{A}_{q-1}^{r}=\mathcal{A}_{q}^{r}=\mathcal{A}_{q+1}^{r}$, (23) can be expressed as

$$
\begin{aligned}
\sigma_{l q}^{2}= & 0.5 N_{0}+\sum_{u \in \mathcal{A}_{q}^{r}}\left(\left|\Re\left(\mathbf{H}_{l q} \mathbf{B}_{u q}\right)\right|^{2}+\right. \\
& 0.646\left|\Im\left(\mathbf{H}_{l q} \mathbf{B}_{u q}\right)\right|^{2}+0.1769\left|\Im\left(\mathbf{H}_{l q-1} \mathbf{B}_{u q-1}\right)\right|^{2} \\
& \left.+0.1769\left|\Im\left(\mathbf{H}_{l q+1} \mathbf{B}_{u q+1}\right)\right|^{2}\right)
\end{aligned}
$$

If the channel frequency selectivity is not severe we can assume that

$$
\left|\Im\left(\mathbf{H}_{l q-1} \mathbf{B}_{u q-1}\right)\right|^{2},\left|\Im\left(\mathbf{H}_{l q+1} \mathbf{B}_{u q+1}\right)\right|^{2} \approx\left|\Im\left(\mathbf{H}_{l q} \mathbf{B}_{u q}\right)\right|^{2}
$$

Then, it follows that (24) can be approximated by

$$
\sigma_{l q}^{2} \approx 0.5 N_{0}+\sum_{u \in \mathcal{A}_{q}^{r}}\left|\mathbf{H}_{l q} \mathbf{B}_{u q}\right|^{2} .
$$

When the subset $K_{1}$ is addressed $\sigma_{l q}^{2}=0.5 N_{0}$. In the general form the rate is given by

$$
\begin{gathered}
r_{l q}=\log _{2}\left(1+S I N R_{l q}\right) \\
S I N R_{l q}=p_{l q}\left\|\mathbf{H}_{l q} \check{\mathbf{U}}_{l q}^{0}\right\|_{2}^{2} / \sigma_{l q}^{2}=p_{l q} \beta_{l q} .
\end{gathered}
$$

Once the interference is updated, the problem associated to the subset $K_{r}$ is

$$
\begin{aligned}
P_{r}: & \underset{\left\{p_{l i}\right\},\left\{\rho_{l i}\right\}}{\operatorname{argmin}} \sum_{l \in K_{r}} \sum_{i=1}^{X}\left|\bar{S}_{i}\right| p_{l i} \\
\text { s.t. } & \sum_{i=1}^{X} \rho_{l i}\left|\bar{S}_{i}\right| \log _{2}\left(1+p_{l i} \hat{\beta}_{l i}\right) \geq R_{l}, \quad l \in K_{r} \\
& \sum_{l \in K_{r}} \rho_{l i} \leq 1, \quad \rho_{l i} \in\{0,1\}, \quad 1 \leq i \leq X,
\end{aligned}
$$

where

$$
\hat{\beta}_{l i}=\left(\prod_{m \in \bar{S}_{i}} \beta_{l m}\right)^{1 /\left|\bar{S}_{i}\right|} .
$$

Similarly to problem (17), the users' rate constraints and the objective function have been defined taking into account that the power within each block is constant. The approximation made in (25) results in (28), which exactly coincides with the SINR that is obtained in OFDM when the sequential channel assignment approach is implemented [6]. Hence, the solution of (29) can be indistinctly used in OFDM and FBMC/OQAM systems. As it has been mentioned we propose to solve (29) by executing the LPSCA algorithm that is described in [6].

\section{NUMERICAL RESULTS}

This section is devoted to evaluating the user selection and the power allocation algorithm proposed in Section V. Regarding the system parameters, the bandwidth is $10 \mathrm{MHz}$, the number of subcarriers is $M=1024$ and the sampling frequency is set to $15.36 \mathrm{MHz}$ so that the subcarrier spacing is $\Delta_{f}=15 \mathrm{KHz}$. The number of users that are connected to the BS is equal to $N_{U}=10$ and they are uniformly distributed in a cell of radius $R=500 \mathrm{~m}$. Since the number of transmit antennas is set to $N_{T}=2$, only two users can be associated with each subcarrier. The thermal noise density is $-174 \mathrm{dBm} / \mathrm{Hz}$ and the channel is modeled as a Rayleigh fading process with a power delay profile that follows the extended pedestrian A (EPA) channel model [11]. The path loss exponent is $\gamma=4$. It should be mentioned that the frequency selectivity of the EPA channel is such that the system model described in Section II is valid. In other words, the channel frequency response can be assumed flat at the subcarrier level. Concerning the air-interface, we consider FBMC/OQAM and OFDM with a CP that encompasses $L_{C P}=M / 14$ samples. To comply with the recommendations proposed by the Technical Specification Group for Radio Access Network of the 3GPP, only 600 out 1024 subcarriers are used to transmit data. Furthermore, subcarriers are gathered in groups of 12 and, thus, there are $X=50$ blocks. In notation terms, let $S_{a}$ denote the set whose elements are the indexes of those subcarriers that are active and $S_{a}(i)$ indicates the $i$ th active subcarrier. Borrowing the notation from Section IV, the elements of subset $\bar{S}_{i}$ when OFDM is implemented are given by $\left\{S_{a}(1+12(i-1)) \ldots S_{a}(12 i)\right\}$, for $i=1, \ldots, 50$. To prevent ICI from degrading the FBMC/OQAM system performance, the blocks are separated by one subcarrier that is intentionally left empty. Then in FBMC/OQAM the number of subcarriers that are able to convey data is extended from 600 to 650 and the subsets are generated as $\bar{S}_{i}=$ $\left\{S_{a}(2+13(i-1)) \ldots S_{a}(13 i)\right\}$, for $i=1, \ldots, 50$. Although inserting one guard band between blocks increases the out of band radiation, the transmitted signal in FBMC/OQAM still fits into the spectrum mask of the Universal Mobile Telecommunication System (UMTS) [12]. Actually, it is shown that the occupied subcarriers can be increased in $10 \%$ without violating the spectrum mask.

The Figure 1 depicts the power that is required to schedule $N_{U}=10$ users in 50 blocks of 12 subcarriers each. As it has been pointed out in Section IV, one subcarrier is left empty between blocks when the FBMC/OQAM 


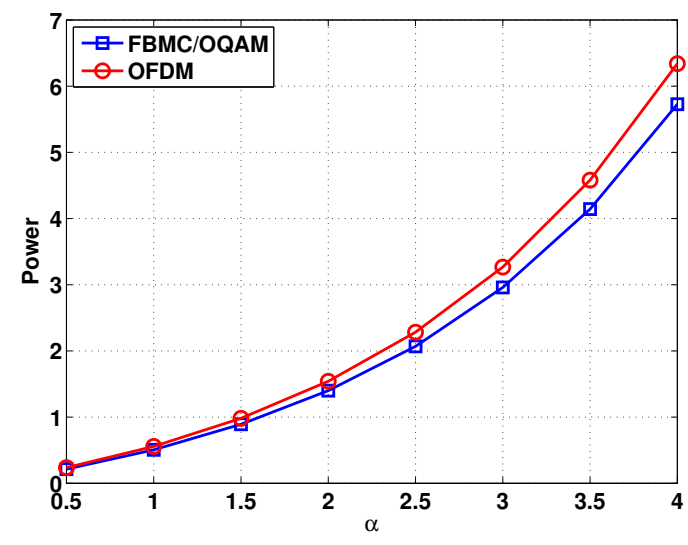

Figure 1. Power vs. spectral efficiency in OFDM and FBMC/OQAM.

modulation is employed. The metric that is evaluated is $\sum_{l=1}^{N_{U}} \sum_{i=1}^{50} 12\left(1+L_{C P} / M\right) 2 p_{l i}$, where the power coefficients $\left\{p_{l i}\right\}$ have been obtained after solving (29). The rate constraints have been set equal for all users as follows: $R_{l}=\alpha 600 / N_{U}$, where $\alpha$ is the target spectral efficiency. It is important to recall that $L_{C P}=0$ in FBMC/OQAM systems, and because of that the transmitted power is reduced when compared to OFDM, as Figure 1 highlights.

In order to verify that both modulations are able to achieve similar rates we have depicted in Figure 2 the overall rate, which is defined as $\sum_{l=1}^{N_{U}} \sum_{q \in S_{a}} r_{l q}$. To this end we have used the exact rate definition given by (27). Nevertheless, the power of the residual interference plus noise in OFDM and FBMC/OQAM systems is characterized by (26) and (24), respectively. In the light of the results of Figure 2 we can conclude that the assumption made in (25) does not have any negative impact as the overall rates achieved in both modulation schemes practically coincide. Another important aspect that is worth highlighting is that the relative difference between the exact sum-rate and the lower bound based on the geometric mean, which is formulated in (15), does not exceed $0.25 \%$ after solving (29). This holds true for OFDM and FBMC/OQAM, which supports the simplifications proposed in this paper. For the sake of the clarity in the presentation of the results the lower bound has not been included in Figure 2.

\section{CONCLUSIONS}

This paper shows that the joint optimization of transmit and receive beamforming, the channel assignment and the power allocation is very challenging in the FBMC/OQAM context. The main reason stems from the fact that the received signals are subject to inter-user, inter-carrier and inter-symbol interference. By grouping subcarriers and keeping the same user selection and power allocation in each group we can exploit spatial diversity to allocate several users in the same frequency resources. It has been demonstrated that the margin adaptive problem in OFDM and FBMC/OQAM systems can be sub-optimally solved resorting to the LPSCA method. Since no energy is wasted in the FBMC/OQAM modulation scheme, this modulation is able to transmit the same amount of information as OFDM but using less power.

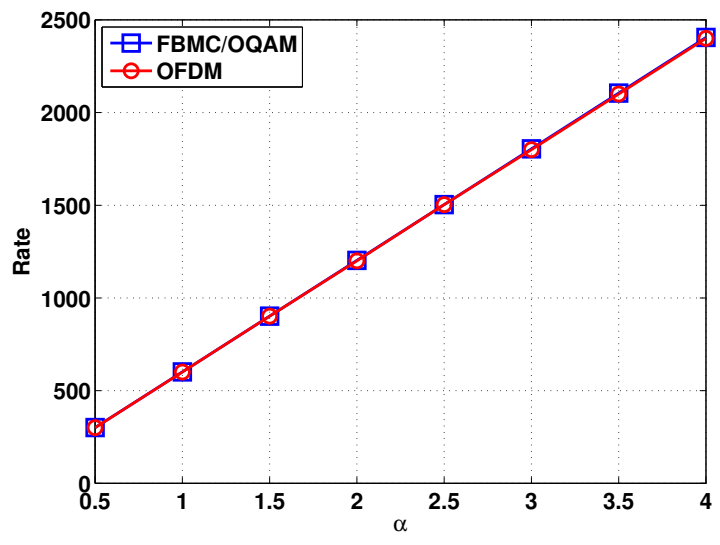

Figure 2. Overall rate vs. spectral efficiency in OFDM and FBMC/OQAM.

\section{ACKNOWLEDGMENT}

This work has received funding from the Spanish Ministry of Economy and Competitiveness (Ministerio de Economia y Competitividad) under project TEC2011-29006-C03-02 (GRE3N-LINKMAC) and from the Catalan Government (2009SGR0891). This work was also supported by the European Commission in the framework of the FP7 Network of Excellence in Wireless COMmunications NEWCOM\# (Grant agreement no. 318306).

\section{REFERENCES}

[1] P. Siohan, C. Siclet, and N. Lacaille, "Analysis and design of OFDM/OQAM systems based on filterbank theory," Signal Processing, IEEE Transactions on, vol. 50, no. 5, pp. 1170 -1183, may 2002.

[2] B. Farhang-Boroujeny, "OFDM Versus Filter Bank Multicarrier," Signal Processing Magazine, IEEE, vol. 28, no. 3, pp. 92 -112, may 2011.

[3] M. Caus, "MIMO Designs for filter bank multicarrier and multiantenna systems based on OQAM," Ph.D. dissertation, Universitat Politecnica de Catalunya (UPC), 2013. [Online]. Available: http://theses.eurasip.org

[4] M. Payaro, A. Pascual-Iserte, A. Garcia-Armada, and M. SanchezFernandez, "Resource Allocation in Multi-Antenna MAC Networks: FBMC vs OFDM," in Vehicular Technology Conference (VTC Spring), 2011 IEEE 73rd, May 2011, pp. 1-5.

[5] M. Shaat and F. Bader, "Computationally Efficient Power Allocation Algorithm in Multicarrier-Based Cognitive Radio Networks: OFDM and FBMC Systems," EURASIP JASP, vol. 2010.

[6] M. Moretti and A. I. Perez-Neira., "Efficient Margin Adaptive Scheduling for MIMO-OFDMA Systems," Wireless Communications, IEEE Transactions on, vol. 12, no. 1, pp. 278-287, 2013.

[7] M. Caus, A. I. Perez-Neira, and M. Moretti, "SDMA for FBMC with block diagonalization," in Signal Processing Advances in Wireless Communications (SPAWC), IEEE 14th International Workshop on, 2013.

[8] M. Bellanger, "Specification and design of a prototype filter for filter bank based multicarrier transmission." ICASSP, 2001, pp. 2417-2420.

[9] Q. H. Spencer, A. L. Swindlehurst, and M. Haardt, "Zero-forcing methods for downlink spatial multiplexing in multiuser MIMO channels," Signal Processing, IEEE Transactions on, vol. 52, no. 2, Feb. 2004.

[10] J. Huang, V. Subramanian, R. Agrawal, and R. Berry, "Downlink scheduling and resource allocation for OFDM systems," Wireless Communications, IEEE Transactions on, vol. 8, no. 1, pp. 288-296, 2009.

[11] 3GPP TS 36.101 V11.8.0, "Evolved Universal Terrestrial Radio Access (E-UTRA); User Equipment (UE) radio transmission and reception (Release 11)," 2014.

[12] L. G. Baltar, D. S. Waldhauser, and J. A. Nossek, "Out-of-band radiation in multicarrier systems: a comparison," in Multi-Carrier Spread Spectrum 2007, ser. Lecture Notes Electrical Engineering. Springer Netherlands, 2007, vol. 1, pp. 107-116. 